\title{
Treating Neurological Wilson's Disease; The Expert Opinion is not Good Enough
}

Can J Neurol Sci. 2013; 40: 445

Wilson's disease (WD) is a rare disorder of copper metabolism. In Canada few neurologists see sufficient patients to become confident in managing and treating this disorder. Patients can present with liver and/or neuropsychiatric symptoms and so these few patients are spread across a number of disciplines including pediatric, liver or neurological clinics. There is a paucity of randomized controlled trials (RCTs) and Evidence Based Medicine reviews for helping such physicians treat WD; thus making it a further challenge to determine the most effective option for an individual patient.

Rana and colleagues present a view point ${ }^{1}$ arguing that zinc should be the preferred drug for treating WD. The authors set out two scenarios, with illustrative cases, related to the use of the commercially available treatments; penicillamine, trientine and zinc. The first scenario is that the clinical symptoms are due to copper deposition in hepatic or brain tissue and the chelators, penicillamine or trientine reduce tissue copper levels by increasing excretion in the urine. However, worsening of neurological symptoms with penicillamine and trientine (although less common) is a significant problem. The second scenario is that WD is due to high circulating levels of copper. Thus use of zinc to bind dietary copper and prevents GI absorption with reduced serum free copper levels will improve symptoms without neurological worsening.

Traditionally the commonest treatment strategy has been to use penicillamine, or more recently trientine, to achieve a rapid de-coppering and reversal of symptoms. Slower de-coppering over months is then achieved by using zinc for either maintenance (started with or after the chelators) or as monotherapy for asymptomatic carriers. Case series with long term follow-ups have generally supported this strategy. Thus use of zinc alone, as suggested by this view point, is less common due to the slower rate of de-coppering and the concern that this delay will worsen symptoms. The counter argument is that zinc has increased safety while achieving equal efficacy. However no RCTs have compared the use or timing of these agents, or the combination of chelation with zinc. Ongoing arguments between experts will continue until more definitive, objective studies are performed. There is a European database prospectively collecting data (www.eurowilson.org) and the North American WD Association http://www.wilsonsdisease.org/ supports ongoing research efforts.

Of concern however is that published case-series continue to show that only a proportion of WD patients with neurological symptoms fully recover (where symptoms are carefully evaluated and reported) (e.g. $\left.{ }^{2-4}\right)$. This suggests that such patients are either not optimally managed or treated, and there is a need for better therapies for the neurological symptoms. This appears to be in contrast to liver disease in WD which responds better to drugs and liver transplant can be curative. Genetic studies may lead to better treatments but there remain many unknown issues, including how to manage the patient with rapidly deteriorating neurological function. In the modern era this seems a travesty and an urgent need for the WD community to address.

Susan Fox

Movement Disorder Clinic, Toronto Western Hospital Toronto,Ontario, Canada.Email: sfox@uhnresearch.ca

\section{REFERENCES}

1. Rana AQ, Avan A, Aftab A, Manssor W, Hoogenraad TU. A viewpoint about the treatment of Wilson's disease. Can J Neurol Sci. 2013;40(4)612-14.

2. Merle U, Schaefer M, Ferenci P, Stremmel W. Clinical presentation, diagnosis and long-term outcome of Wilson's disease: a cohort study. Gut. 2007 Jan;56(1):115-20.

3. Taly AB, Meenakshi-Sundaram S, Sinha S, Swamy HS, Arunodaya GR. Wilson disease: description of 282 patients evaluated over 3 decades. Medicine (Baltimore). 2007 Mar;86(2):112-21.

4. Moores A, Fox SH, Lang AE, Hirschfield G. Wilson disease: Canadian perspectives on presentation and outcomes from an adult ambulatory setting. Can J Gastroenterol. 2012 Jun;26(6): $333-9$. 